\title{
Tuning Solvent Miscibility: A Fundamental Assessment on the Example of Induced Methanol $/ n$-Dodecane Phase Separation
}

\author{
Roberto Macchieraldo, ${ }^{\dagger}$ Sascha Gehrke, ${ }^{\dagger,+}$ Nagaphani K. Batchu, ${ }^{\S \odot}$ Barbara Kirchner, ${ }^{*}, \oplus$ \\ and Koen Binnemans, ${ }^{*}, \odot$ \\ ${ }^{\dagger}$ Mulliken Center for Theoretical Chemistry, University of Bonn, Beringstrasse 4+6, D-53115 Bonn, Germany \\ ${ }^{\ddagger}$ Max Planck Institute for Chemical Energy Conversion, Stiftstrasse 34-36, D-45413 Mülheim an der Ruhr, Germany \\ ${ }^{\S}$ Department of Chemistry, KU Leuven, Celestijnenlaan 200F, bus 2404, B-3001 Heverlee, Belgium
}

Supporting Information

\begin{abstract}
In this work, we assess the fundamental aspects of mutual miscibility of solvents by studying the mixing of two potential candidates, methanol and $n$-dodecane, for nonaqueous solvent extraction. To do so, ${ }^{1} \mathrm{H}$ NMR spectroscopy and molecular dynamics simulations are used jointly. The NMR spectra show that good phase separation can be obtained by adding $\mathrm{LiCl}$ and that the addition of a popular extractant (tri-n-butyl phosphate) yields the opposite effect. It is also demonstrated that in a specific case the poor phase separation is not due to the migration of $n$-dodecane into the more polar phase, but due to the transfer of the extractant into it, which is especially relevant when considering industrial applications of solvent extraction. With the aid of molecular dynamics simulations, explanations of this behavior are given. Specifically, an increase of all hydrogen-bond lifetimes is found to be consequent to the addition of $\mathrm{LiCl}$ which implies an indirect influence on the methanol liquid structure, by favoring a stronger hydrogen-bond network. Therefore, we found that better phase separation is not directly due to the presence of $\mathrm{LiCl}$, but due to the "hardening" of the hydrogen-bond network.
\end{abstract}

\section{INTRODUCTION}

Nowadays, hydrometallurgical approaches, which involve aqueous chemistry for the recovery of metals, are fundamental for the extractive metallurgy of many elements. The protocols for the extraction of many metal ions are based on a combination of pyrometallurgy, which consists of thermal treatments aimed to produce physical and chemical transformations, and hydrometallurgy. The latter is often used in the first part of a flow sheet. ${ }^{1-3}$ Unfortunately, pyrometallurgical methods are usually incapable of treating low-grade ores or residues in an economic way and hydrometallurgy with strong acid leaching is weakly selective. These problems, together with the aim to develop and establish a circular economy, ${ }^{4}$ led to the innovative concept of solvometallurgy. ${ }^{5}$

Solvometallurgy implies processes that are similar to those of hydrometallurgy, but not involving aqueous phases. This opens a wide spectrum of choices, including molecular organic solvents, ionic liquids, deep-eutectic solvents, and inorganic solvents. $^{6-12}$ It must be clarified that the term "nonaqueous solvents" in the paradigm of solvometallurgy does not necessarily imply anhydrous conditions, but rather a solvent in which the water content is lower than 50 vol \%. Solvometallurgy has already been exploited for the recovery of copper from chrysocolla, ${ }^{13,14}$ rare earth and other metals from complex silica-rich ores, ${ }^{15}$ uranium from carbonate ores and for reprocessing of spent nuclear fuel. ${ }^{16}$ In hydrometallurgy, conventional solvent extraction is commonly used. In this case, metals are distributed between an aqueous phase and an immiscible organic phase. As mentioned above, the new paradigm of solvometallurgy replaces the aqueous phase by a nonaqueous solvent. ${ }^{17,18}$ Additionally, some conditions have to be fulfilled: (1) the process should involve two mutually immiscible liquid phases, (2) phase separation should be fast, (3) good solubility of the extractant in the less polar phase, (4) good solubility of the extracted metal complex, and (5) poor solubility of the extractant in the more polar phase. The first condition is crucial, especially with regard to industrial applications. Hence, enormous effort is made in the identification of suitable solvent pairs for nonaqueous solvent extraction. The first indication of mutual miscibility is a volume change of two phases that are in contact with each other. This does not hold if the mutual solubility is the same in the two phases. Therefore, the mutual solubility can be quantified for instance by Fourier transform infrared spectroscopy, gas chromatography, or NMR spectroscopy. Furthermore, mutual miscibility is a function of the temperature and the concentration of cosolvents, which can be a dissolved salt or extractant. For example, it is known that two completely miscible solvents can be phase-separated with the addition of salt to the more polar phase. ${ }^{19,20}$ The opposite is also possible, that is, the addition of extractants to the less polar phase can worsen the phase separation by causing the transfer of the solvent molecules from one phase to the other.

Received: January 28, 2019

Revised: April 13, 2019

Published: April 29, 2019 
In miscibility experiments, two candidates are mixed together and the phase separation is evaluated. To improve the phase separation, the addition of salts can be taken into account. When the obtained phase separation is satisfactory, the extractant is added and the evaluation is repeated. The addition of the extractant can be considered as a critical step since for many systems it leads to unsuccessful phase separation.

In this paper, we aim to highlight those fundamental aspects that act as a driving force to the mutual solubility of two solvents in the presence of cosolvents and cosolutes and, therefore, have to be taken into account while screening for new solvent pairs. To do this, ${ }^{1} \mathrm{H}$ NMR spectroscopy and molecular dynamics (MD) simulations are used to obtain a fundamental insight into the mixing and demixing of two potential candidate solvents for solvent extractions, such as methanol $(\mathrm{MeOH})$ and $n$-dodecane (DD). ${ }^{21-23}$ Quantitative analysis experiments were performed and based on these results, several systems were simulated (see Figure 1) to

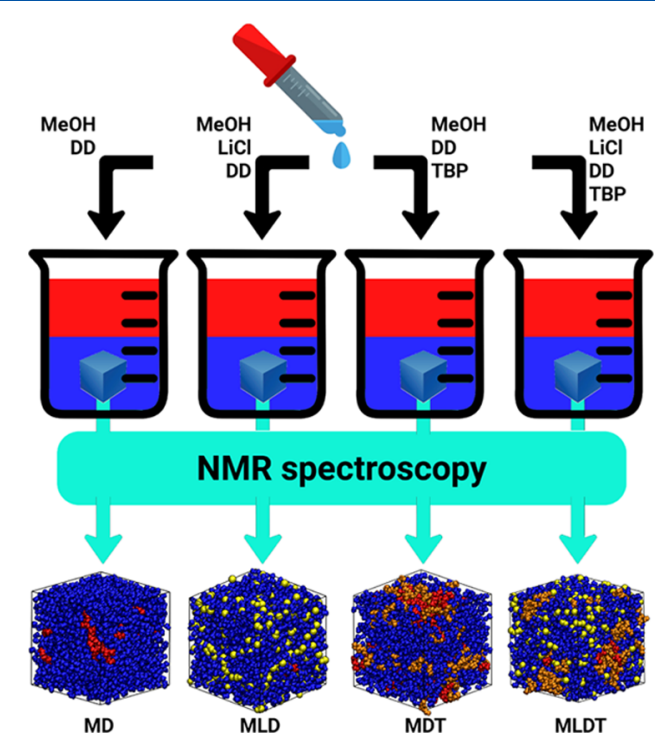

Figure 1. Schematic representation of the procedure described in this work.

provide molecular-level insight into the polar phase obtained after the mixing and demixing of the two solvents. The system was also tested and simulated in the presence of $\mathrm{LiCl}$ and the popular solvating extractant tri- $n$-butyl phosphate (TBP).

\section{METHODS}

Chemicals and Instrumentation. Methanol (>99.9\%), $n$ dodecane (>99\%), and acetone- $d_{6}(99.9$ atom \% D) were purchased from Acros Organics (Geel, Belgium) while TBP (>98\%) was purchased from Alfa-Aesar (Karlsruhe, Germany). Chloroform-d (99.8 atom \% D) and $\mathrm{LiCl}$ (99.9\%) were obtained from Sigma-Aldrich (Diegem, Belgium). The mixing was performed using an Eppendorf ThermoMixer C, in $\mathrm{T}$ Corning Centrifuge Tubes with CentriSTAR caps. Centrifugation was performed by means of a Heraeus Megafuge 1.0. ${ }^{1} \mathrm{H}$ NMR spectra were recorded on a Bruker Avance 300 spectrometer, operating at $300 \mathrm{MHz}$. The chemical shifts are noted in parts per million (ppm), referenced to tetramethylsilane. All chemicals were used as received without any further purification.
Experimental Details. Solutions of $\mathrm{MeOH}$ with different concentrations $\left(0,0.5,1,2\right.$, and $\left.3 \mathrm{~mol} \cdot \mathrm{L}^{-1}\right)$ of $\mathrm{LiCl}$ were prepared and stirred overnight. $5 \mathrm{~mL}$ of each solution was dispensed in centrifuge tubes and mixed with $5 \mathrm{~mL}$ of DD or $\mathrm{DD}+\mathrm{TBP}\left(1 \mathrm{~mol} \cdot \mathrm{L}^{-1}\right)$. The centrifuge tubes were shaken for $30 \mathrm{~min}$ at $2000 \mathrm{rpm}$ and centrifuged for $15 \mathrm{~min}$ at $4000 \mathrm{rpm}$. The volume changes of the phases were evaluated using the indicated graduation of the centrifuge tubes. The same procedure was then repeated with a fixed concentration of $\mathrm{LiCl}\left(2 \mathrm{~mol} \cdot \mathrm{L}^{-1}\right)$ and by varying the concentration of TBP $\left(0.25,0.5,1,2 \mathrm{~mol} \cdot \mathrm{L}^{-1}\right)$. After each step, solutions were left to rest to reach thermal equilibrium with the environment.

${ }^{1} \mathrm{H}$ NMR spectra of the different phases were collected. The amount of dissolved solvent and in some cases also the amount of extractant were quantified using the ratio of the integrated peak values belonging to different molecules. To do so, two NMR solvents were used: deuterated acetone (acetone- $d_{6}$ ) and deuterated chloroform (chloroform- $d_{1}$ ). The NMR solvent should not strongly interact with any compound to avoid interference. Deuterated acetone was found to have an effect on the $\mathrm{MeOH}$ acidic hydrogen atom peak. Nevertheless, the integration has been performed on the peaks of the methyl group hydrogen atoms, therefore, these spectra provide the qualitative and quantitative information we were looking for. With the aim of testing the effect of a different number of scans and different relaxation times on the final spectra, the data were collected with different number of scans $(8-32)$ and different relaxation times $(20-40 \mathrm{~s})$. All spectra are in good agreement with each other, validating the gathered data.

Computational Details. The initial configuration of the simulation boxes was generated using PACKMOL (version 17.039). ${ }^{24}$ Classical molecular dynamics simulations were performed using the LAMMPS program package (version 17 Nov 2016). ${ }^{25}$ For $\mathrm{MeOH}, \mathrm{DD}$, and $\mathrm{LiCl}$, the well-known OPLS-AA force field were used, ${ }^{26}$ whereas for TBP we opted for the force field recently developed by Ali et al. ${ }^{27}$ since it was proven to perform very well when TBP is mixed with $n$ dodecane. Nonbonded interactions were described by the 612 Lennard-Jones potential. ${ }^{28}$ Lorentz-Berthelot mixing rules were used to obtain parameters for pairs of different atoms. A cutoff of $1.6 \mathrm{~nm}$ was selected for the calculation of LennardJones and Coulombic interactions and a particle-particle particle-mesh solver, mapping the atom charge to a threedimensional mesh. ${ }^{29}$ Equilibration of the systems was obtained by simulating for $0.5 \mathrm{~ns}$ using the NPT ensemble. Constant pressure and temperature were achieved applying the NoséHoover chain thermostat and barostat $(T=297.15 \mathrm{~K}, \tau=100$ fs and $P=1$ bar, $\tau=1000 \mathrm{fs}$, respectively). ${ }^{30,31}$ The cell vectors, averaged over the last 250 ps were taken to perform the production run within the NVT ensemble. The production run consisted of $10 \mathrm{~ns}$ of the simulation time after $0.5 \mathrm{ps}$ of equilibration. The time step was set to $0.5 \mathrm{fs}$ during the whole procedure. The obtained trajectories were analyzed with the TRAVIS code. ${ }^{32}$ This tool offers different kinds of functions allowing the analysis of the interaction among the components of the systems. Intra- and intermolecular interactions can be taken into account. In this work radial distribution functions (RDFs), Voronoi analysis, mean square displacements (MSDs), and hydrogen-bond lifetime calculations were exploited. ${ }^{33-37}$ 


\section{RESULTS}

Volume Analysis. As mentioned in the Introduction, one of the key conditions for two solvents to be suitable for solvent extraction is the formation of two immiscible liquid phases. In this respect, the most immediate test is to check the volume change of the solvents, after mixing and phase separation. Volume changes which are not significant in a laboratory workflow might result in a critical solvent loss on an industrial scale. The optimal situation is when no volume change occurs. The observed volume changes of the phases are reported in Table 1. Numbers are referring to the volume ratio between

Table 1. Volume Ratios upon Mixing Equal Volumes of $\mathrm{MeOH}$ and DD (at Room Temperature) with Respect to Different Concentrations $\left(\mathrm{mol} \cdot \mathrm{L}^{-1}\right)$ of $\mathrm{LiCl}$ in $\mathrm{MeOH}$ and TBP (always $1 \mathrm{~mol} \cdot \mathrm{L}^{-1}$ ) in $\mathrm{DD}^{a}$

$\begin{array}{llc}\mathrm{MeOH}+\mathrm{LiCl} & \mathrm{DD} & \mathrm{DD}+\mathrm{TBP} \\ \text { neat } \mathrm{MeOH} & 1: 1.1 & 1: 2.3 \\ 0.5 & 1: 1 & 1: 2 \\ 1 & 1: 1 & 1: 1.9 \\ 2 & 1: 1 & 1: 1.9 \\ 3 & 1: 1 & 1: 1.9\end{array}$

${ }^{a}$ Data are referred to the volume ratio between the top phase (apolar) and the bottom phase (polar) obtained after mixing and phase separation.

the top phase (apolar) and the bottom phase (polar). The values reported in Table 1 show that the neat binary system $\mathrm{MeOH}+\mathrm{DD}$ is not suitable for solvent extraction. Increasing the concentration of $\mathrm{LiCl}$ improved phase separation. An optimal phase ratio was already obtained for $0.5 \mathrm{~mol} \cdot \mathrm{L}^{-1}$ of this salt. Yet, Table 1 shows that with $1 \mathrm{~mol} \cdot \mathrm{L}^{-1}$ of TBP the phase separation worsened and that even with higher concentrations of $\mathrm{LiCl}$ (up to $3 \mathrm{~mol} \cdot \mathrm{L}^{-1}$ ) it was not possible to obtain the 1:1 ratio. In Table 2 the volume ratios are

Table 2. Volume Ratios with Respect to Different Concentrations $\left(\mathrm{mol} \cdot \mathrm{L}^{-1}\right)$ of the Extractant for $\mathrm{MeOH}+$ $\mathrm{LiCl} 2 \mathrm{~mol} \cdot \mathrm{L}^{-1}$ a

$\begin{array}{lc}\mathrm{DD}+\mathrm{TBP} & \mathrm{MeOH}+\mathrm{LiCl} \\ \text { neat DD } & 1: 1 \\ 0.25 & 1: 1.4 \\ 0.5 & 1: 1.8 \\ 1 & 1: 1.9 \\ 2 & 1: 4.5\end{array}$

${ }^{a}$ Data reported is referred to the volume ratio between the top phase (apolar) and the bottom phase (polar) after mixing and phase separation.

reported for a fixed concentration of $\mathrm{LiCl}\left(2 \mathrm{~mol} \cdot \mathrm{L}^{-1}\right)$, and the TBP concentration ranging from 0 to $2 \mathrm{~mol} \cdot \mathrm{L}^{-1}$. Again, in the presence of TBP, the optimal phase separation could not be achieved.

${ }^{1} \mathrm{H}$ NMR Spectra. We used ${ }^{1} \mathrm{H}$ NMR spectroscopy to evaluate the amount of $\mathrm{DD}$ and $\mathrm{MeOH}$ being transferred from one phase to the other. Figures S1-S4 (see the Supporting Information (SI)) show the spectra of the polar (bottom) phase which were obtained after shaking and centrifuging of, respectively, $\mathrm{MeOH}$ and $\mathrm{DD}(\mathrm{MD}), \mathrm{MD}$ in the presence of TBP (MDT), MD in the presence of $\mathrm{LiCl}$ (MLD), and MD in the presence of both TBP and $\mathrm{LiCl}$ (MLDT). The evaluation of the ratio of the integrals of specific peaks allowed to estimate the ratio of the number of molecules of $\mathrm{MeOH}, \mathrm{DD}$, and TBP in the polar phase. For the MD system, the $\mathrm{MeOH} / \mathrm{DD}$ ratio was 92. With the addition of up to $3 \mathrm{~mol} \cdot \mathrm{L}^{-1} \mathrm{LiCl}$, the situation radically changed, moving the ratio to 1840 . In the case of MDT, the ratio was 30, whereas for MLDT it was 300 . The following conclusion can be drawn: the addition of $\mathrm{LiCl}$ prevents the transfer of the DD into the polar phase, whereas TBP facilitates it. Moreover, the volume change is not only due to the transfer of DD into the bottom phase but also due to the transfer of TBP. In fact, the $\mathrm{MeOH} / \mathrm{DD}$ ratios are not fully in line with what we expected from the phase volume analysis. Specifically, the "quality" of the phase separation of these systems according to the volume analysis is MLD > MD > MLDT > MDT, whereas according to the $\mathrm{MeOH} / \mathrm{DD}$ ratios in NMR spectra, we have MLD > MLDT > MD > MDT. The explanation may be found in the $\mathrm{MeOH} / \mathrm{TBP}$ ratios, which remain stable even in the presence of $\mathrm{LiCl}$. Therefore, MLDT shows a worse phase separation than $\mathrm{MD}$, despite the fact that the amount of DD in the polar phase is much lower. To align the volume ratio analysis with the NMR results, we can count TBP together with $\mathrm{DD}$ and recalculate the $\mathrm{MeOH} /(\mathrm{DD}+$ TBP) ratios for all systems. We finally obtain $\operatorname{MLD}(1838)>$ $\operatorname{MD}(92)>\operatorname{MLDT}(23)>\operatorname{MDT}(13)$, which is in line with the volume analysis.

Systems Simulated via Molecular Dynamics. Four systems were simulated in accordance with the evidence gathered from the ${ }^{1} \mathrm{H}$ NMR spectra (see Figure 1), namely, the bottom phases that were obtained after the mixing and phase separation of the $\mathrm{MeOH}+\mathrm{DD}$ mixture $(\mathrm{MD})$ also in the presence of TBP (MDT), $\mathrm{LiCl}$ (MLD), and both (MLDT). Table 3 reports the number of molecules in each system.

Table 3. Compositions of Simulated Bottom Phase Systems Chosen According to ${ }^{1} \mathrm{H}$ NMR Spectra ${ }^{a}$

$\begin{array}{lrrrr}\text { system } & \text { MD } & \text { MDT } & \text { MLD } & \text { MLDT } \\ \text { MeOH } & 3000 & 3000 & 3000 & 3000 \\ \text { DD } & 32 & 100 & 2 & 10 \\ \text { TBP } & & 120 & & 120 \\ \text { LiCl } & & & 361 & 361\end{array}$

${ }^{a}$ Data refer to the number of molecules in each system.

Solvent Partitioning. By means of the Voronoi tessellation method, a domain analysis was performed. ${ }^{38-40}$ With this analysis, we divided the systems into subsets of different molecules or groups of atoms and calculated the average number of domains of each subset during the simulation. A large value means the subset is dispersed, whereas a small value means the atoms belonging to the subset are all connected. We decided to divide the systems into subsets of molecules of the same kind, and also to pair $\mathrm{MeOH}$ $+\mathrm{LiCl}$ and $\mathrm{DD}+\mathrm{TBP}$. The results of these different analyses are reported in Table 4. A value close to one means high aggregation. Large values indicate small dispersed agglomerates.

In the $\mathrm{MD}$ system, $\mathrm{MeOH}$ forms one single domain, most probably due to the large excess of this solvent. The 32 molecules of DD form an average of 12.9 domains, which means that DD molecules are clustering in groups of 2-3 molecules into $\mathrm{MeOH}$.

In MLD system, $\mathrm{MeOH}$ again forms one single domain and $\mathrm{LiCl}$ is well solvated by $\mathrm{MeOH}$ itself (in fact the subset $\mathrm{MeOH}$ 
Table 4. Bottom Phases Domain Analysis ${ }^{a}$

\begin{tabular}{|c|c|c|c|c|c|c|}
\hline system & $\mathrm{MeOH}$ & $\mathrm{MeOH}+\mathrm{LiCl}$ & $\mathrm{LiCl}$ & $\mathrm{DD}$ & $\mathrm{DD}+\mathrm{TBP}$ & ТВР \\
\hline $\mathrm{MD}$ & 1.0 & & & $12.9(32)$ & & \\
\hline MLD & 1.0 & 1.0 & $291.8(361)$ & $1.5(2)$ & & \\
\hline MDT & 2.7 & & & $9.3(100)$ & 3.9 & $6.4(120)$ \\
\hline MLDT & 1.2 & 1.2 & $274.0(361)$ & $7.0(10)$ & 3.4 & $3.6(120)$ \\
\hline
\end{tabular}

${ }^{a_{T}}$ The values refer to the average number of domains of the subsets into the systems (within parentheses is the number of molecules for that component. For $\mathrm{MeOH}$, the number of molecules is always 3000).

$+\mathrm{LiCl}$ forms one domain, while $\mathrm{LiCl}$ is divided into 291.8 domains, corresponding to an average of $2.4 \mathrm{Li} / \mathrm{Cl}$ per domain). According to the ${ }^{1} \mathrm{H}$ NMR spectra, in the presence of $\mathrm{LiCl}$, to reproduce the experimental concentration only two molecules of DD were allowed; these molecules form an average of 1.5 domains, which means that they were clustered together for half of the simulation.

In the MDT system, the total number of DD molecules was 100 , which can be related to the activity of TBP. In this case, $\mathrm{MeOH}$ is divided into 2.7 domains, which is reasonable once we take into account the amount of DD and TBP in the system. DD forms 9.3 domains (meaning clusters of 10-11 molecules) and TBP 6.4 (clusters of 20-21 molecules). Taking into account the number of domains for the DD + TBP subset, and comparing it to the individual DD and TBP, we find a reduction to 3.9 domains. This shows that DD and TBP cluster together, and consequently "stabilize" each other.

This is even more evident in the MLDT system. For this system, the number of domains for the subsets $\mathrm{MeOH}$ and $\mathrm{MeOH}+\mathrm{LiCl}$ is 1.2 , due to the smaller amount of DD and TBP. In this case, TBP forms 3.6 domains, showing the negative influence of $\mathrm{LiCl}$ in the solvation of TBP by $\mathrm{MeOH}$. On the other hand, the 10 molecules of DD form 7 domains, which is misleading since it might seem that in this system DD can be well solvated. Analysis of the DD + TBP subset is especially handy in this case. In fact, for this subset, we find an average number of domains very close to the one of the TBP subset. Hence, we can draw the conclusion that DD has to be fully solvated by TBP to transfer into the polar phase. This is also shown from the snapshot reported in Figure 2, where DD is completely surrounded by TBP and $\mathrm{LiCl}$ is surrounded by $\mathrm{MeOH}$.

Mobility. The dynamics of the systems were studied by calculating the mean square displacement (MSD) and consequently the diffusion constant of the different components (see Figure 3 and Table 5). The MSDs in Figure 3 allow an easy comparison of the mobility of different compounds in the systems.

We observed that $\mathrm{MeOH}$ is mainly affected by the presence of $\mathrm{LiCl}$, which lowers its mobility (increasing the total viscosity of the system) to half of the values for pure methanol. TBP also slightly decreases the mobility of $\mathrm{MeOH}$, which is in line with the fact that TBP and $\mathrm{MeOH}$ can interact via hydrogen bonds, and since TBP is a bulky molecule (with low mobility), it decreases the mobility of $\mathrm{MeOH}$ as a consequence.

$\mathrm{DD}$ is more mobile in the MD system, where the lack of strong interactions with TBP and the small amount of DD itself (which prevents a strong clustering with itself) allows DD to move "freely". The lower mobility of DD in the MDT and MLDT systems can be easily attributed to the clustering of DD with itself or with TBP. Again, the higher viscosities in the MLD and MLDT systems (due to $\mathrm{LiCl}$ ) strongly influence the results. With respect to the addition of TBP, MSDs of $\mathrm{Li}^{+}$and

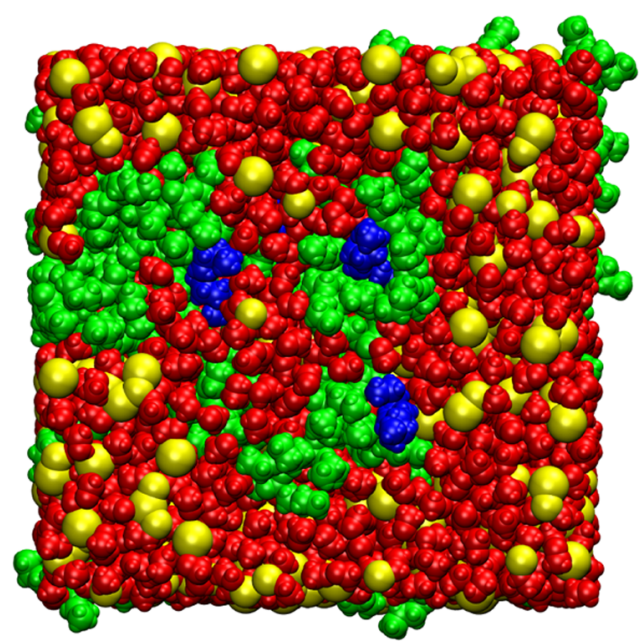

Figure 2. Snapshot taken from the MLDT system. All atoms are represented by their van der Waals radii. Color scheme: yellow for $\mathrm{LiCl}$, red for $\mathrm{MeOH}$, green for TBP, and blue for DD.

$\mathrm{Cl}^{-}$seem to comply with those of $\mathrm{MeOH}$ by decreasing accordingly, pointing to a correlation between them. Interestingly, $\mathrm{Cl}^{-}$is slightly faster than $\mathrm{Li}^{+}$.

To allow some further considerations, we plotted the MSDs of TBP and DD in the MDT and MLDT systems. The curves show that the dynamics of these molecules might be related. Yet, it is clear that TBP is more influenced by the addition of $\mathrm{LiCl}$ than DD.

Table 5 shows the diffusion coefficients of the components in different systems. The experimental diffusion coefficient of neat $\mathrm{MeOH}$ is $2.6 \times 10^{-9} \mathrm{~m}^{2} \cdot \mathrm{s}^{-1}$, and in our simulation of the system MD, we obtained $2.5 \times 10^{-9} \mathrm{~m}^{2} \cdot \mathrm{s}^{-1}$, which is in very good agreement. We see that in all systems, $\mathrm{MeOH}$ molecules are more mobile than $\mathrm{DD}$, the ions and TBP. It is also interesting that the dynamics of the ions are only slightly influenced by the presence of TBP.

Structural Properties. We used radial pair distribution functions (RDFs) to further interpret the data gathered in the previous sections. Figure 4 displays the RDFs of the interplay between $\mathrm{MeOH}$ molecules and $\mathrm{Li}^{+}$. With respect to the $\mathrm{MeOH}-\mathrm{MeOH}$ interactions, the MDT system shows the highest value first peak. The second highest value of the first peak is from the MD system. Interestingly, MLD and MLDT systems show the smallest first peak. This is easy to explain once we take into account the shoulder of both curves at 340 $\mathrm{pm}$. These shoulders are only visible for systems which contain $\mathrm{LiCl}$ and therefore, they are due to the proximity of $\mathrm{MeOH}$ molecules that are interacting with the same $\mathrm{LiCl}$ ion. ${ }^{41}$ It is reasonable to conclude that because of the strong interaction between $\mathrm{MeOH}$ and $\mathrm{LiCl}$, the ions interpose between $\mathrm{MeOH}$ molecules, creating an even stronger network (showing the kosmotropic character of $\mathrm{LiCl}$ ), as shown by the increase in 

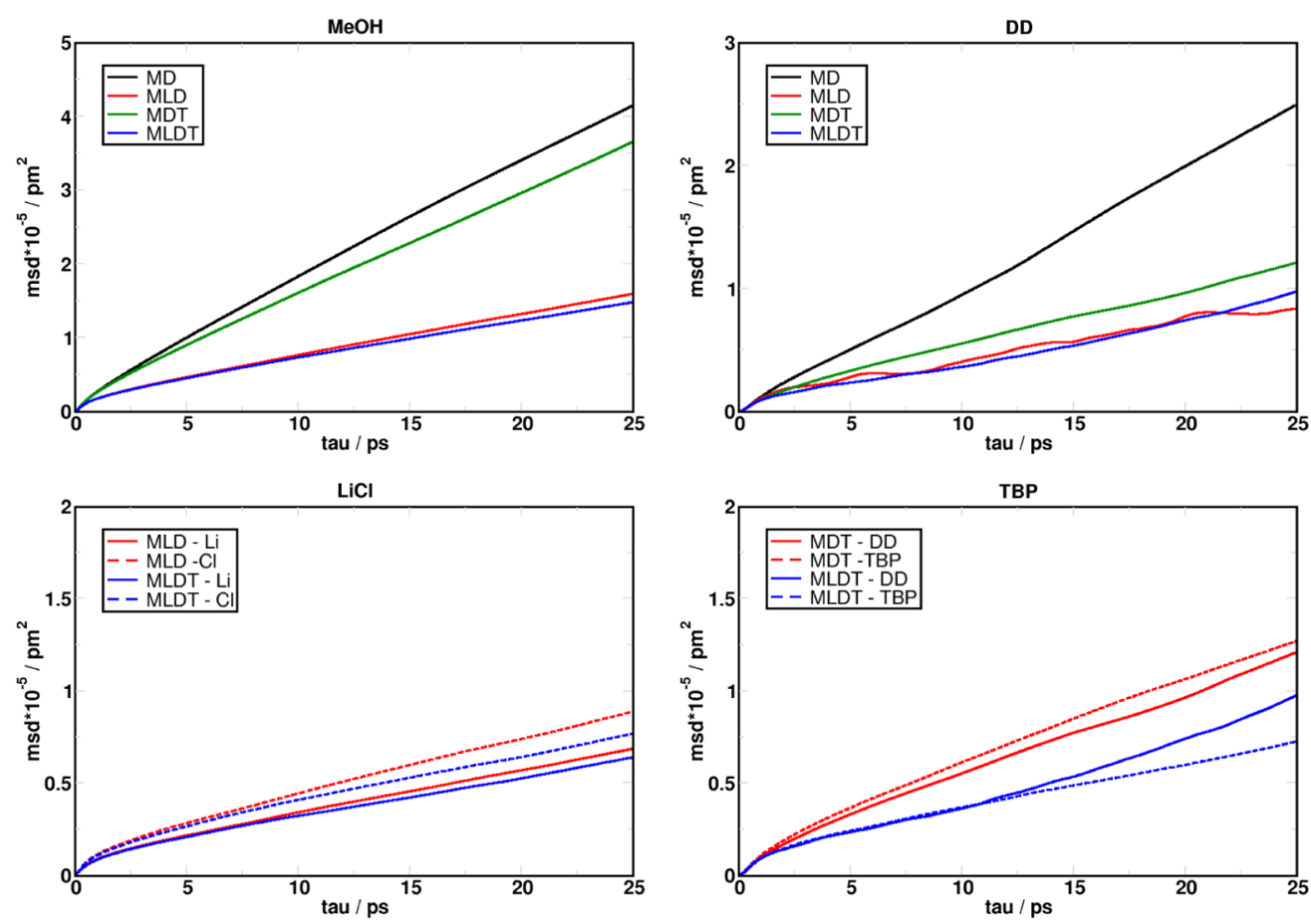

Figure 3. Mean square displacements of the bottom phase components. Top left: $\mathrm{MeOH}$; top right: DD; bottom left: $\mathrm{Li}^{+}$and $\mathrm{Cl}^{-}$; bottom right: $\mathrm{DD}$ and TBP.

Table 5. Diffusion Coefficient $\left(\times 10^{-9}, \mathrm{~m}^{2} \cdot \mathrm{s}^{-1}\right)$ in the Bottom Phases

$\begin{array}{lccccc}\text { component } & \mathrm{MeOH} & \mathrm{Li}^{+} & \mathrm{Cl}^{-} & \mathrm{DD} & \mathrm{TBP} \\ \text { MD } & 2.53 & & & 1.74 & \\ \text { MLD } & 0.91 & 0.38 & 0.48 & 0.47 & \\ \text { MDT } & 2.27 & & & 0.71 & 0.71 \\ \text { MLDT } & 0.82 & 0.36 & 0.39 & 0.69 & 0.39\end{array}$

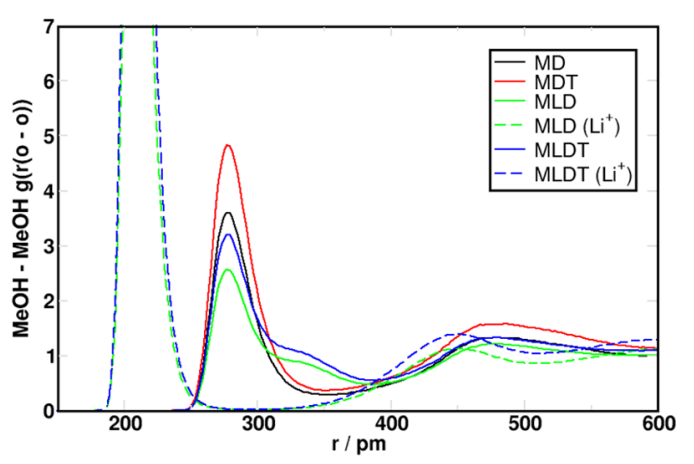

Figure 4. RDFs of the interactions of $\mathrm{MeOH}$ molecules in the bottom phases.

viscosity in these systems. This is also confirmed by the highest peaks in Figure 4, which represent the interactions between $\mathrm{MeOH}$ molecules and $\mathrm{Li}^{+}$. Figure 5 shows a snapshot of the aforementioned situation. It is clear that $\mathrm{MeOH}$ interacts with $\mathrm{Li}^{+}$and $\mathrm{Cl}^{-}$via the oxygen and the hydrogen atoms, respectively. Furthermore, it is possible to notice some hydrogen bonds between $\mathrm{MeOH}$ molecules, a topic which we tackle in the next section.

In Figure 6, we present an intramolecular analysis of the RDFs of the two terminal carbons of DD. With the aid of this analysis, we could define when DD is more crumpled or stretched out in different systems. In the picture, we identified

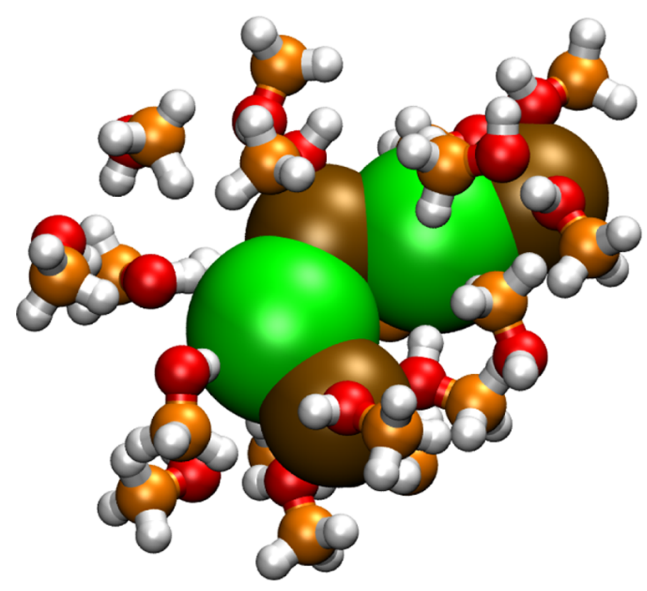

Figure 5. Example of the liquid structure of $\mathrm{MeOH}$ in the presence of $\mathrm{LiCl}$ taken from the MLDT system. $\mathrm{LiCl}$ is represented with van der Waals radii while $\mathrm{MeOH}$ is displayed with the ball-and-stick models. Color scheme: brown for $\mathrm{Li}$, green for $\mathrm{Cl}$, orange for $\mathrm{C}$, red for $\mathrm{O}$, and white for $\mathrm{H}$.

three distances for which the molecules seem more stabilized: (1) $400 \mathrm{pm}$, which implies a crumpled structure, (2) $1050 \mathrm{pm}$, which implies a stretched out structure, and (3) $850 \mathrm{pm}$, which is the half-way case. In the MD system, the crumpled structure is almost equally relevant as the stretched one, whereas the addition of TBP in MDT and MLDT systems induces a relaxation of the structure toward the more stretched one. The comparison between MDT and MLDT systems confirmed once more the stabilizing effect of TBP on DD. In fact, in these systems, the amount of TBP was the same while having 100 DD molecules in the MDT system and only 10 DD molecules in the MLDT system. Consequently, it is reasonable that in the MLDT system, DD is completely surrounded by TBP, which allows the full relaxation of DD. In the MDT system, due to 


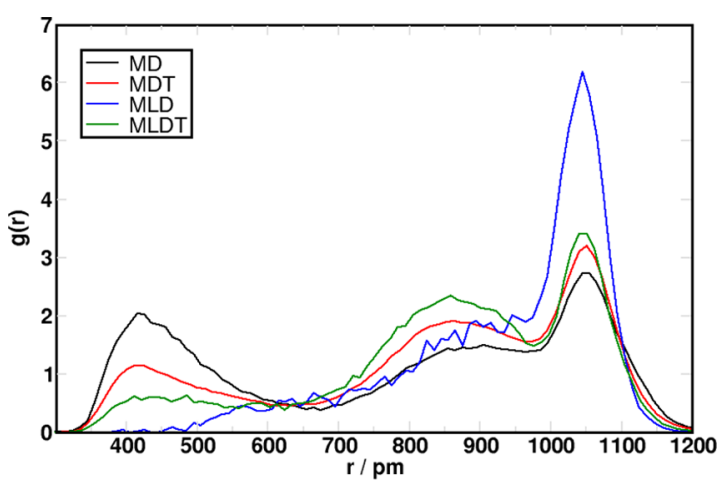

Figure 6. Intramolecular RDFs of the distance between the terminal carbons of DD molecules.

this larger amount of DD, TBP may not be able to interpose completely between $\mathrm{DD}$ and $\mathrm{MeOH}$ due to the larger amount of DD, which leads to a more crumpled structure of DD. For the sake of completeness, we also reported the analysis for the MLD system. However, since this system contains only two molecules of DD, the statistical sampling for this specific analysis is too poor and does not allow to draw any conclusion.

Hydrogen-Bond (HB) Network. Following the observations of the previous section, we decided to calculate the average hydrogen-bond (HB) lifetimes in our systems (see Table 6), to confirm the effect of $\mathrm{LiCl}$ on the solvent liquid

Table 6. Average Lifetime (ps) of the Hydrogen Bond between $\mathrm{MeOH}$ (Donor) and the Components of Different Systems

\begin{tabular}{lccc} 
& $\mathrm{MeOH}$ & $\mathrm{Cl}^{-}$ & TBP \\
MD & 13.8 & & \\
MLD & 28.8 & 15.5 & \\
MDT & 16.5 & & 10.2 \\
MLDT & 32.6 & 18.7 & 20.1 \\
\hline
\end{tabular}

structure. This was possible by means of the methodology introduced by Luzar and Chandler. ${ }^{34-37}$ The $\mathrm{HB}$ donor is always the $\mathrm{MeOH}$ oxygen atom and depending on the system, we have different acceptors: $\mathrm{MeOH}(\mathrm{O}), \operatorname{LiCl}\left(\mathrm{Cl}^{-}\right)$, and $\mathrm{TBP}\left(\mathrm{O}^{\mathrm{sp} 2}\right)$. By comparing the $\mathrm{HB}$ dynamics for $\mathrm{MeOH}-$ $\mathrm{MeOH}$ in different systems, we can evaluate the influence of other compounds and extend our molecular-level understanding of the systems.

We see that the addition of $\mathrm{LiCl}$ strongly slows down the $\mathrm{HB}$ dynamics for $\mathrm{MeOH}-\mathrm{MeOH}$. This observation is crucial as it shows that $\mathrm{LiCl}$ has a sort of "pinning effect" on $\mathrm{MeOH}$. This pinning effect organizes and stabilizes the $\mathrm{MeOH}$ liquid structure by slowing down the $\mathrm{HB}$ dynamics without hindering the $\mathrm{HB}$ formation (Figure 5). Since the presence of $\mathrm{LiCl}$ is clearly related to the lower miscibility of DD in $\mathrm{MeOH}$, we can easily correlate the slower, therefore stronger, $\mathrm{HB}$ network to the lower miscibility. In fact, the diffusional motion of the hydrophobic solute molecule can be explained via the diffusion of a void containing the molecule itself. This diffusion process becomes less likely with such a slow $\mathrm{HB}$ dynamics, ${ }^{42}$ which explains the lower miscibility. Interestingly, TBP slows down the dynamics of $\mathrm{MeOH}-\mathrm{MeOH} \mathrm{HB}$. On the other hand, since the interaction between TBP and $\mathrm{MeOH}$ (shown by the RDFs analysis in the SI) is weaker, we cannot draw the same conclusion we drew for $\mathrm{LiCl}$. An easy explanation for the slower dynamics in the presence of TBP is that the dissolution of any molecule, be it hydrophobic or hydrophilic, tends to reduce solvent entropy in hydrogen-bonding solvents. Therefore, DD and TBP influence on the HB network agrees with previous studies on the effect of hydrophobic molecules on the solvent HBs. ${ }^{43-46}$ Another effect to keep in mind, which further justifies our findings, is the effect of secondary alkyl groups on the HB formation energetics. ${ }^{47}$

\section{CONCLUSIONS}

By means of a comprehensive approach, which includes experimental data and theoretical simulations, we studied the fundamental aspects of solvent miscibility. We investigated the mixing of two potential candidate solvents (methanol and $n$ dodecane) for solvent extraction, in the paradigm of solvometallurgy. Experimentally, we found that good separation of the two solvents can be achieved by the addition of a salt (in this case $\mathrm{LiCl}$ ) and that the addition of a popular extractant appeared to worsen the phase separation. We also highlighted, by means of ${ }^{1} \mathrm{H}$ NMR spectroscopy, in the case of the full MLDT system, that poor phase separation is not due to the migration of $n$-dodecane into the more polar phase, but rather due to the migration of tri- $n$-butyl phosphate into the polar phase. This is an interesting result, especially for industrial applications of nonaqueous solvent extraction.

The "polar" phases obtained during the experimental study were investigated by means of classical molecular dynamics simulations. We found that $n$-dodecane tends to cluster into methanol and tends to dissolve into tri- $n$-butyl phosphate microphase. $\mathrm{LiCl}$ exhibited a greater impact with respect to the overall dynamics. In fact, we observed an increase of the viscosity, which we correlated to an increase of the hydrogenbond lifetimes. Consequently, the addition of $\mathrm{LiCl}$ not only implied an addition of strong interactions between different components of the system, but also an indirect influence on the liquid structure of methanol, by favoring a stronger hydrogen-bond network. This may be the reason why experimentally we have found that the amount of $n$-dodecane in the bottom phase of the MLDT system is lower than in the MDT system, with the amount of tri- $n$-butyl phosphate being the same, since tri- $n$-butyl phosphate can be part of the hydrogen-bond network, and consequently the exclusion of $n$ dodecane is not due to the direct presence of $\mathrm{LiCl}$, but due to the "hardening" of the hydrogen-bond network. This effect recalls what had been previously observed by Jiang et al. ${ }^{48}$ while studying the roles of the hydrophobic effect and hydrogen bonding in systems able to selectively recognize hydrophilic molecules in water. Following the concept that it is not the addition of the salt that directly improves the phase separation, but rather a consequence of the hydrogen-bond network, new ways of improving phase separation of two solvents can be thought and tested. For example, it was proven that the local structure of water reorganizes in the vicinity of polyelectrolyte brushes, leading to an enhancement of the hydrogen-bond network. ${ }^{49}$ Furthermore, a similar effect on the methanol liquid structure can be obtained by playing with temperature and pressure. ${ }^{50}$ This kind of effect might be exploited similarly to how $\mathrm{LiCl}$ has been used in our work.

\section{ASSOCIATED CONTENT}

\section{Supporting Information}

The Supporting Information is available free of charge on the ACS Publications website at DOI: 10.1021/acs.jpcb.9b00839. 
${ }^{1} \mathrm{H}$ NMR spectra; radial distribution function; RDFs of the interactions of $\mathrm{MeOH}(\mathrm{O})$ molecules (PDF)

\section{AUTHOR INFORMATION}

\section{Corresponding Authors}

*E-mail: kirchner@thch.uni-bonn.de (B.K.).

*E-mail: Koen.Binnemans@kuleuven.be (K.B.).

ORCID $\odot$

Nagaphani K. Batchu: 0000-0002-1971-6661

Barbara Kirchner: 0000-0001-8843-7132

Koen Binnemans: 0000-0003-4768-3606

Notes

The authors declare no competing financial interest.

\section{ACKNOWLEDGMENTS}

B.K., K.B., and R.M. acknowledge the MSCA ETN project SOCRATES (http://etn-socrates.eu/), which has received funding from the European Union's EU Framework Program for Research and Innovation Horizon 2020 under Grant Agreement No. 721385. S.G. was supported by the International Max Planck Research School for Reactive Structure Analyses for Chemical Reactions. K.B. and N.K.B. acknowledge funding from the European Research Council (ERC) under the European Union's Horizon 2020 Research and Innovation Program: Grant Agreement 694078-Solvometallurgy for critical metals (SOLCRIMET).

\section{REFERENCES}

(1) Hiskey, B. Kirk-Othmer Encyclopedia of Chemical Technology; Wiley Blackwell: Hoboken, New Jersey, 2000.

(2) Anderson, C. G.; Dunne, R. C.; Uhrie, J. L. Mineral Processing and Extractive Metallurgy: 100 years of Innovation; Society for Mining, Metallurgy, and Exploration: Englewood, 2014.

(3) Hayes, P. C. Process Principles in Minerals and Materials Production; Hayes Publishing Co: Sherwood, 2003.

(4) Geissdoerfer, M.; Savaget, P.; Bocken, N. M.; Hultink, E. J. The circular economy - a new sustainability paradigm? J. Clean. Prod. 2017, 143, 757-768.

(5) Binnemans, K.; Jones, P. T. Solvometallurgy: an emerging branch of extractive netallurgy. J. Sustainable Metall. 2017, 3, 570600.

(6) Abbott, A. P.; Boothby, D.; Capper, G.; Davies, D. L.; Rasheed, R. K. Deep eutectic solvents formed between choline chloride and carboxylic acids: versatile alternatives to ionic liquids. J. Am. Chem. Soc. 2004, 126, 9142-9147.

(7) Rogers, R. D.; Seddon, K. R. Ionic liquids-solvents of the future? Science 2003, 302, 792-793.

(8) Gutowski, K. E.; Broker, G. A.; Willauer, H. D.; Huddleston, J. G.; Swatloski, R. P.; Holbrey, J. D.; Rogers, R. D. Controlling the aqueous miscibility of ionic liquids: aqueous biphasic systems of Weter-miscible ionic liquids and water-structuring salts for recycle, metathesis, and separations. J. Am. Chem. Soc. 2003, 125, 6632-6633.

(9) Earle, M. J.; Esperança, J. M.; Gilea, M. A.; Lopes, J. N. C.; Rebelo, L. P.; Magee, J. W.; Seddon, K. R.; Widegren, J. A. The distillation and volatility of ionic liquids. Nature 2006, 439, 831-834.

(10) Fukumoto, K.; Yoshizawa, M.; Ohno, H. Room temperature ionic liquids from 20 Natural amino acids. J. Am. Chem. Soc. 2005, 127, 2398-2399.

(11) Wang, Y.; Voth, G. A. Unique spatial heterogeneity in ionic liquids. J. Am. Chem. Soc. 2005, 127, 12192-12193.

(12) Scurto, A. M.; Aki, S. N. V. K.; Brennecke, J. F. CO2 as a separation switch for ionic liquid/organic mixtures. J. Am. Chem. Soc. 2002, 124, 10276-10277.

(13) Sastri, V. Selective leaching of metals from ores with organic solvents and chelating agents. J. Sci. Ind. Res. 1975, 34, 663-665.
(14) Raghavan, S.; Fuerstenau, D. A Lyometallurgical Process for Leaching Copper from Chrysocolla. In Hydrometallurgy Fundamentals, Technology and Innovations, Proceedings of the Milton E., Wordsworth (IV) Int. Symposium on Hydrometallurgy, 1993; pp 283-297.

(15) Chizhevskaya, S. V.; Chekmarev, A. M.; Klimenko, O. M.; Povetkina, M. V.; Sinegribova, O. A.; Cox, M. In Nontraditional Methods of Treating High-Silicon Ores Containing Rare Elements, Hydrometallurgy '94: Papers presented at the international symposium 'Hydrometallurgy ' 94 ' organized by the Institution of Mining and Metallurgy and the Society of Chemical Industry, and held in Cambridge, England, from 11 to 15 July, 1994; pp 219-228.

(16) Hiroshi, T.; Kazushige, T.; Yoshimi, O. Direct Dissolution of Water-Insoluble Uranium Compounds by Contact with Neutral Organic Solvents Pretreated with Nitric Acid. U.S. Patent US3288568, 1966.

(17) Batchu, N. K.; Vander Hoogerstraete, T.; Banerjee, D.; Binnemans, K. Non-aqueous solvent extraction of rare-earth nitrates from ethylene glycol to n-dodecane by Cyanex 923. Sep. Purif. Technol. 2017, 174, 544-553.

(18) Li, Z.; Li, X.; Raiguel, S.; Binnemans, K. Separation of transition metals from rare earths by non-aqueous solvent extraction from ethylene glycol solutions using Aliquat 336. Sep. Purif. Technol. 2018, 201, 318-326.

(19) Misawa, M.; Yoshida, K.; Maruyama, K.; Munemura, H.; Hosokawa, Y. Salt-induced phase separation in aqueous solution. J. Phys. Chem. Solids 1999, 60, 1301-1306.

(20) Batchu, N. K.; Vander Hoogerstraete, T.; Banerjee, D.; Binnemans, K. Separation of rare-earth ions from ethylene glycol $(+\mathrm{LiCl})$ solutions by non-aqueous solvent extraction with Cyanex 923. RSC Adv. 2017, 7, 45351-45362.

(21) Casás, L. M.; Touriño, A.; Orge, B.; Marino, G.; Iglesias, M.; Tojo, J. Thermophysical properties of acetone or methanol $+\mathrm{n}-$ alkane (C9 to C12) mixtures. J. Chem. Eng. Data 2002, 47, 887-893.

(22) Casás, L. M.; Orge, B.; Díaz, C.; Tojo, J. Liquid-liquid equilibria for mixtures of methyl acetate+methanol+n-alkane $(\mathrm{C} 10-\mathrm{C} 12)$ at several temperatures and $1 \mathrm{~atm}$. J. Chem. Thermodyn. 2004, 36, 237243.

(23) Touriño, A.; Casás, L.; Marino, G.; Iglesias, M.; Orge, B.; Tojo, J. Liquid phase behaviour and thermodynamics of acetone+methanol +n-alkane (C9-C12) mixtures. Fluid Phase Equilib. 2003, 206, 6185 .

(24) Martínez, L.; Andrade, R.; Birgin, E. G.; Martínez, J. M. A package for building initial configurations for molecular dynamics simulations. J. Comput. Chem. 2009, 30, 2157-2164.

(25) Plimpton, S. Fast parallel algorithms for short-range molecular dynamics. J. Comput. Phys. 1995, 117, 1-19.

(26) Jorgensen, W. L.; Maxwell, D. S.; Tirado-Rives, J. Development and testing of the OPLS all-atom force field on conformational energetics and properties of organic liquids. J. Am. Chem. Soc. 1996, $118,11225-11236$.

(27) Das, A.; Sahu, P.; Ali, S. M. Molecular dynamics simulation for the calibration of the OPLS force field using DFT derived partial charges for the screening of alkyl phosphate ligands by studying structure, dynamics, and thermodynamics. J. Chem. Eng. Data 2017, $62,2280-2295$.

(28) Lennard-Jones, J. E. Cohesion. Proc. Phys. Soc. 1931, 43, 461482.

(29) Eastwood, J.; Hockney, R.; Lawrence, D. P3M3DP-The threedimensional periodic particle-particle/particle-mesh program. Comput. Phys. Commun. 1984, 35, 618-619.

(30) Nosé, S. Constant-temperature molecular dynamics. J. Phys.: Condens. Matter 1990, 2, SA115.

(31) Hoover, W. G.; Posch, H. A.; Holian, B. L.; Gillan, M. J.; Mareschal, M.; Massobrio, C. Dissipative irreversibility from Nosé's reversible mechanics. Mol. Simul. 1987, 1, 79-86.

(32) Brehm, M.; Kirchner, B. TRAVIS - A free analyzer and visualizer for monte carlo and molecular dynamics trajectories. $J$. Chem. Inf. Model. 2011, 51, 2007-2023. 
(33) Brehm, M.; Weber, H.; Thomas, M.; Hollòczki, O.; Kirchner, B. Domain analysis in nanostructured liquids: A post-molecular dynamics study at the example of ionic liquids. ChemPhysChem 2015, $16,3271-3277$.

(34) Luzar, A.; Chandler, D. Effect of environment on hydrogen bond dynamics in liquid water. Phys. Rev. Lett. 1996, 76, 928-931.

(35) Luzar, A.; Chandler, D. Hydrogen-bond kinetics in liquid water. Nature 1996, 379, 55-57.

(36) Luzar, A. Resolving the hydrogen bond dynamics conundrum. J. Chem. Phys. 2000, 113, 10663-10675.

(37) Gehrke, S.; von Domaros, M.; Clark, R.; Hollòczki, O.; Brehm, M.; Welton, T.; Luzar, A.; Kirchner, B. Structure and lifetimes in ionic liquids and their mixtures. Faraday Discuss. 2018, 206, 219-245.

(38) Brehm, M.; Weber, H.; Thomas, M.; Hollòczki, O.; Kirchner, B. Domain analysis in nanostructured liquids: a post-molecular dynamics study at the example of ionic liquids. ChemPhysChem 2015, $16,3271-3277$.

(39) Elfgen, R.; Hollòczki, O.; Kirchner, B. A molecular level understanding of template effects in ionic liquids. Acc. Chem. Res. 2017, 50, 2949-2957.

(40) Macchieraldo, R.; Esser, L.; Elfgen, R.; Voepel, P.; Zahn, S.; Smarsly, B. M.; Kirchner, B. Hydrophilic ionic liquid mixtures of weakly and strongly coordinating anions with and without water. ACS Omega 2018, 3, 8567-8582.

(41) Ray, P.; Vogl, T.; Balducci, A.; Kirchner, B. Structural investigations on lithium-doped protic and aprotic ionic liquids. $J$. Phys. Chem. B 2017, 121, 5279-5292.

(42) Kirchner, B.; Hutter, J.; Kuo, I.-F. W.; Mundy, C. J. Hydrophobic hydratation from Car-Parrinello simulations. Int. J. Mod. Phys. B 2004, 18, 1951-1962.

(43) Ludwig, R. Water: From Clusters to the Bulk. Angew. Chem., Int. Ed. 2001, 40, 1808-1827.

(44) Huber, H.; Kirchner, B.; Searles, D. J. Is there an iceberg effect in the water/DMSO mixture? Some information from computational chemistry. J. Mol. Liq. 2002, 98-99, 71-77.

(45) Galamba, N. Water's structure around hydrophobic solutes and the iceberg model. J. Phys. Chem. B 2013, 117, 2153-2159.

(46) Chowdhary, J.; Ladanyi, B. M. Hydrogen bond dynamics at the water/hydrocarbon interface. J. Phys. Chem. B 2009, 113, 4045-4053.

(47) Li, Q.; Wu, G.; Yu, Z. The role of methyl groups in the formation of hydrogen bond in DMSO-methanol mixtures. J. Am. Chem. Soc. 2006, 128, 1438-1439.

(48) Yao, H.; Ke, H.; Zhang, X.; Pan, S.-J.; Li, M.-S.; Yang, L.-P.; Schreckenbach, G.; Jiang, W. Molecular recognition of hydrophilic molecules in water by combining the hydrophobic effect with hydrogen bonding. J. Am. Chem. Soc. 2018, 140, 13466-13477.

(49) Yamazoe, K.; Higaki, Y.; Inutsuka, Y.; Miyawaki, J.; Cui, Y.-T.; Takahara, A.; Harada, Y. Enhancement of the hydrogen-bonding network of water confined in a polyelectrolyte brush. Langmuir 2017, 33, 3954-3959.

(50) Wallen, S. L.; Palmer, B. J.; Garrett, B. C.; Yonker, C. R. Density and temperature effects on the hydrogen bond structure of liquid methanol. J. Phys. Chem. 1996, 100, 3959-3964. 